\title{
Investigation of Physical Triplet Quenching by Electron Donors
}

\author{
Ulrich Stelner, * Gerhard Winter, and Horst E. A. Kramer
}

Institut für Physikallsche Chemle der Universität Stuttgart, Pfaffenwaldring 55, D-7000 Stuttgart 80, West Germany

\begin{abstract}
Absolute radical yields, as determined by flash spectroscopy, are reported for the reaction of the thionine triplet ${ }^{3} \mathrm{TH}^{+}$and its protonated form ${ }^{3} \mathrm{TH}_{2}{ }^{2+}$ with a variety of electron donors in methanolic solution. The yields are extrapolated to infinite donor concentrations. Two methods of obtaining absolute yields are described. The different yields with different donors are due to partial physical quenching of the triplets in the electron transfer reaction. The results are discussed in terms of different models, capable of explaining bimolecular intersystem crossing processes. A triplet exciplex as an intermediate in the electron transfer reaction is favored.
\end{abstract}

\section{Introduction}

For the study of fast electron transfer processes the use of electronically excited species is most convenient, as they can be rapidly produced by short excitation pulses and are more reactive than the ground state molecules, the electron affinity, or ionization potential respectively, being more favorable for the electron transfer reaction by the amount of the excitation energy.

Since the first experimental proof by Leonhardt and Weller ${ }^{1}$ of radical formation in the fluorescence quenching of perylene by amines in polar solvents, electron transfer reactions with excited singlet states have been extensively studied. ${ }^{2}$ In general the quantum yield of radical formation is quite low even under conditions of complete fluorescence quenching and does not exceed absolute values of $0.5 .^{3}$ This is mainly due to the possibility of the originating radical pair to undergo fast recombination to the energetically lower ground state competing with the formation of free radicals by diffusive separation.

Besides radical formation and deactivation to the ground state fast bimolecular triplet formation constitutes a third channel of electronic deactivation in the quenching of excited singlet states by electron donors or acceptors. ${ }^{4}$

For electron transfer reactions of excited triplet states much less experimental information about the mechanism is available than in the singlet case. This is because, due to their lower energy, triplet states are less reactive than the corresponding excited singlet states and in the case of long singlet lifetimes, the triplet reaction cannot be separated from the singlet reaction.

In the case of triplets reacting with electron donors or acceptors in polar solvents there are two reaction channels: electron transfer, giving the radicals, or bimolecular physical quenching giving the ground state molecules. The physical quenching is an intersystem crossing process and from this point of view corresponds to the fast triplet formation in the case of singlet reactions, rather than to the spin conserving ground state deactivation. In a radical pair originating from an overall triplet encounter complex the unpaired electron spins are still in a triplet alignment. Hence a fast geminate recombination to the ground state molecules is spin forbidden and one should expect higher yields of free radicals than in the case of singlet reactions. ${ }^{5}$ In this paper we report the absolute quantum yields of radical formation and physical quenching of the thionine triplet ${ }^{3} \mathrm{TH}^{+}$and its protonated form ${ }^{3} \mathrm{TH}_{2}{ }^{2+}$ in their reactions with a variety of electron donors in methanolic solution, as determined by flash spectroscopy.

We expect that systematic investigations of this kind will improve understanding of the mechanisms of both bimolecular intersystem crossing and electron transfer.
The intermediates of thionine photoreductions have been extensively studied. ${ }^{6-9}$ A list of the intermediates relevant to the present work is given in Table I. Unless the concentrations of the electron donors are very high, the thionine triplet is the primary reacting state. As the lifetime of the first excited singlet state is very short (2.4 $\times 10^{-10} \mathrm{~s}^{6,37}$ ) almost complete triplet quenching can be achieved with many electron donors before singlet quenching is observed.

Acids of different strengths are required to protonate thionine and its photochemical intermediates. Strong acids are needed to protonate the thionine ground state $\mathrm{TH}^{+}$ ( $\mathrm{p} K$ in water is $-0.3^{9}$ ) but the corresponding equilibrium in the triplet state

${ }^{3} \mathrm{TH}^{+}+\mathrm{H}^{+} \overleftarrow{\rightarrow}{ }^{3} \mathrm{TH}_{2}{ }^{2+}$

has a $\mathrm{p} K^{*}$ of 7.0 in methanol. ${ }^{10}$ By the acceptance of an electron the triplet forms are converted to the corresponding forms of semireduced thionine. The equilibrium between the semireduced thionine $\mathrm{TH}$. (basic semithionine) and its protonated form $\mathrm{TH}_{2}{ }^{+}$. (acid semithionine) corresponds to a $\mathrm{p} K^{*}$ of 10.4 in methanol. ${ }^{10}$ The rate constants for electron transfer reactions with the thionine triplets ${ }^{3} \mathrm{TH}^{+}$and ${ }^{3} \mathrm{TH}_{2}{ }^{2+}$, in the following denoted as "basic" and "acid" triplet, are found to be correlated with the free enthalpy of these reactions ${ }^{11}$ analogously to singlet quenching by electron donors as reported by Rehm and Weller. ${ }^{12} \mathrm{As}$ the acid triplet has a higher redox potential than the basic form one can understand its higher reactivity, which is observed especially with donors, which react fairly slowly with the basic triplet. ${ }^{10,13}$

For the study of the quantum yields of radical formation and physical quenching the comparison of the triplet forms ${ }^{3} \mathrm{TH}^{+}$and ${ }^{3} \mathrm{TH}_{2}{ }^{2+}$ appear to be interesting cases of variation of the electron accepting component, as these excited species can be transformed into each other by an adiabatic reaction.

\section{Experimental Section}

The flash apparatus was the same as described by Kramer. ${ }^{14}$ A Kodak Wratten filter No. 15 was inserted between the flash lamp and the photolysis cell to absorb light of wavelengths shorter than $500 \mathrm{~nm}$. The solutions investigated were deaerated by passing $\mathrm{N}_{2}$ (oxygen content lower than $5 \mathrm{ppm}$ ) through them for $0.5 \mathrm{~h}$.

Chemicals. Methanol (Merck p.A.) was used as solvent throughout. Thionine was purified according to Endriss. ${ }^{15}$ The purity of the donor compounds was of the highest commercial grade; additional purification is given in parentheses: 9,10-dimethylanthracene (DMA, EGA, recrystallized twice from ethanol), diazabicyclo[2.2.2]octane 
TABLE I: List of Symbols for Thionine and Its Intermediates

Acid semithionine

(DABCO, EGA), 1,3-diphenylisobenzofurane (EGA), azulene (Fluka), p-bromoaniline (Merck), bromobenzene (Merck, distilled in vacuo), allylthiourea (Merck, twice recrystallized from water), selenourea (Merck, recrystallized from water under an $\mathrm{N}_{2}$ atmosphere), hydroquinone (Merck, recrystallized twice from water under an $\mathrm{N}_{2}$ atmosphere). $p$-Methoxydimethylaniline was synthesized in our laboratory according to the procedures in ref 16 and purified by sublimation. The phosphoric acid was $96 \%$ (Merck p.A.). Buffer solutions of $\mathrm{pH}^{*} 8.6$ in methanol (for definition, see ref 17 ) were prepared according to Broser and Fleischhauer. ${ }^{18}$ The resulting solutions were $0.015 \mathrm{M}$ in phenylacetic acid and $0.005 \mathrm{M}$ in sodium methylate.

Methods. The donor substances were chosen to fulfill the following conditions: Complex formation between donor-acceptor molecules in the ground state should be excluded. This was ensured by checking the additivity of the absorption spectra. With donor concentrations at which reaction with the thionine triplet occurs almost quantitatively, no appreciable singlet quenching should be observed. This was examined by measuring the thionine fluorescence. No donors, whose rate constants with the basic thionine triplet were less than $8 \times 10^{6} \mathrm{M}^{-1} \mathrm{~s}^{-1}$, were investigated in compliance with the latter condition.

For the determination of the yields of the electron transfer reaction

${ }^{3} \mathrm{TH}^{+}+\mathrm{R} \rightarrow \mathrm{TH} \cdot+\mathrm{R}^{+}$.

or

${ }^{3} \mathrm{TH}_{2}{ }^{2+}+\mathrm{R} \rightarrow \mathrm{TH}_{2}^{+}+\mathrm{R}^{+}$

measurements of radical concentrations are preferable to those of concentrations of the reacting molecules, since the percentage changes of the latter are small. The quantity of semithionine produced proved to be a suitable measure for yields with different donors. Due to its strong absorption and the long wavelength position of its absorption band (see Figure 1) the acid form of semithionine $\mathrm{TH}_{2}^{+}$. is more suitable than the basic form TH., since the absorption maxima of the cation radicals of the donors are at shorter wavelengths.

As was shown in an earlier paper, ${ }^{19}$ in unbuffered methanolic solutions electron transfer reactions with the basic thionine triplet ${ }^{3} \mathrm{TH}^{+}$yield the basic semithionine TH. The difference in $\mathrm{p} K^{*}$ between triplet and semithionine makes it possible, however, to find a $\mathrm{pH}^{*}$ at which the basic semithionine is quantitatively protonated, while the basic triplet is not protonated. ${ }^{10}$ Therefore the re- actions of the basic triplet were investigated in methanolic solutions, buffered at $\mathrm{pH}^{*} 8.6$ (the buffer concentration was $0.02 \mathrm{M}$ ). As the protonation is very fast, it is still the reaction of ${ }^{3} \mathrm{TH}^{+}$with the donor $\mathrm{R}$ which determines the rate of formation and the yield of the detected radicals $\mathrm{TH}_{2}^{+}$:

${ }^{3} \mathrm{TH}^{+}+\mathbf{R} \rightarrow\left[\mathrm{TH} \cdot+\mathrm{R}^{+} \cdot \underset{\mathrm{H}^{+}}{\text {fast }}\right] \mathrm{TH}_{2}^{+}+\mathbf{R}^{+}$

To produce the acid triplet we used $0.1 \mathrm{M}$ phosphoric acid in methanol. Under this condition protonation occurs in the triplet state, not in the ground state. Again the protonation is very fast and the reaction of ${ }^{3} \mathrm{TH}_{2}{ }^{2+}$ with the donor is rate determining:

$\left[{ }^{3} \mathrm{TH}^{+}+\mathrm{R} \underset{\mathrm{H}^{+}}{\text {fast }}\right]{ }^{3} \mathrm{TH}_{2}{ }^{2+}+\mathrm{R} \rightarrow \mathrm{TH}_{2}^{++}+\mathrm{R}^{+}$.

The lifetime of the two triplet forms in the presence of the corresponding buffer or acid concentrations, given above, was determined to be $12 \mu \mathrm{s}$ in the absence of any quencher. The lifetime of the semithionine radicals (initial slope of the decay curve, converted into a first-order rate constant-the order of the decay is not uniform) was in all cases longer than $1 \mathrm{~ms}$, so that the yield of semithionine could be measured conveniently after complete triplet reaction.

The molar extinction coefficients of the basic triplet ${ }^{3} \mathrm{TH}^{+}$and the acid semithionine $\mathrm{TH}_{2}{ }^{+}$, , respectively, were determined by simultaneously measuring the optical density changes at 780 and $600 \mathrm{~nm}$ (maximum of ground state absorption). For this purpose the monitoring light beam was split behind the photolysis cell in order to measure the intensity by two monochromator photomultiplier assemblies. Solutions of thionine $(2.5$ to $4 \times$ $\left.10^{-6} \mathrm{M}\right)$ were used. Semithionine was produced by adding $5 \times 10^{-4} \mathrm{M} 9,10$-dimethylanthracene to the solution, buffered at $\mathrm{pH}^{*}$ 8.6. (The absorption of the DMA cation at $780 \mathrm{~nm}$ amounts to less than $3 \%$ of the semithionine absorption, as can be seen if a basic solution containing $5 \times 10^{-5} \mathrm{M}$ sodium methylate is used. In this case only the basic form of the semithionine TH. is formed, which does not absorb at $780 \mathrm{~nm} .^{7}$ Hence the absorption observed is due to the DMA cation radical only. From its absorption at $780 \mathrm{~nm}$ it can be concluded that, according to the optical density spectrum given in ref 20 , the $a b$ sorption at $600 \mathrm{~nm}$ is also negligible relative to the thionine ground state absorption.) 


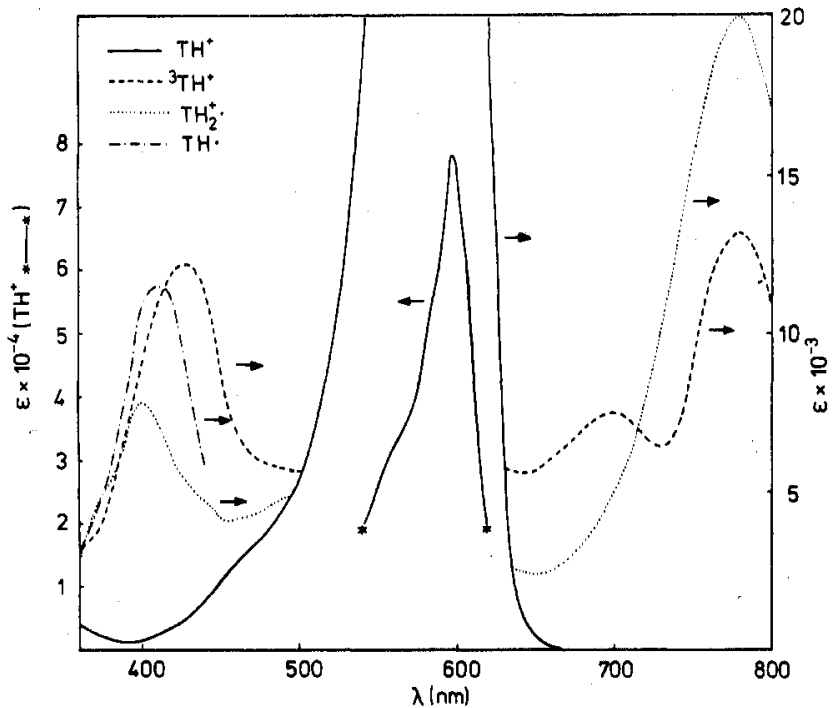

Figure 1. Absorption spectra of thionine ground state $\mathrm{TH}^{+}$, basic triplet ${ }^{3} \mathrm{TH}^{+}$, basic semithionine $\mathrm{TH}$, and acid semithionine $\mathrm{TH}_{2}{ }^{+}$. in methanol. The spectra were taken with dye concentrations of $2 \times 10^{-5}, 6 \times 10^{-6}$, $4 \times 10^{-6}$, and $5 \times 10^{-6} \mathrm{M}$, respectively. The two forms of semithionine were produced by $\mathrm{DABCO}\left(2 \times 10^{-3} \mathrm{M}\right)$, for $\mathrm{TH} \cdot$ the methanol was unbuffered, for $\mathrm{TH}_{2}{ }^{+}$. the buffer had a $\mathrm{pH}^{*}$ of $\mathbf{8 . 6}$ (see Experimental Section). The spectra of the intermediates were taken in oxygen free solutions, they are corrected for ground state absorption.

The concentrations of the intermediates were calculated, assuming that the optical density change at $600 \mathrm{~nm}$ is entirely due to the disappearance of thionine ground state. This yields a lower bound of the concentration of the intermediates and hence an upper bound of their extinction coefficients $\left(\epsilon_{780}\left({ }^{3} \mathrm{TH}^{+}\right)=13200, \epsilon_{780}\left(\mathrm{TH}_{2}{ }^{+} \cdot\right)=\right.$ 20000). The spectra of the intermediates (Figure 1) were measured with higher concentrations of thionine. Therefore accurate measurements in the region of the $600-\mathrm{nm}$ peak of the ground state absorption were not possible. In the spectral region, however, where the absorption of the ground state is comparable to that of the intermediates, the spectra of the intermediates could be obtained by correcting the measured change in optical density for the calculated change in the ground state absorption. If the extinction coefficients used for the intermediates were too high, the corrected spectra should be curved downward in the region of the sharp rise of the ground state absorption. This is not the case (see Figure 1). So we can assume that the extinction coefficients obtained by the procedure described above are fairly accurate.

Evaluation of the Quantum Yield. The concentration of radicals produced by constant flash intensity is proportional to the quantum yield of radical formation only if the concentration of the absorbing ground state does not change appreciably during the flash duration. (In the case of appreciable bleaching during the flash, triplet molecules deactivated to the ground state might become excited again. Such an effect would increase the apparent radical yield of substrates exhibiting strong physical quenching compared to substances with a better radical forming ability.) Flash intensity therefore was kept small, so that not more than $10 \%$ of the thionine molecules were excited by a single flash.

Extrapolation to Infinite Donor Concentration. When evaluating the yield of semithionine produced, one has to take into account that even without adding any electron donor, a certain amount of semithionine is formed. This is presumably due to a partially occurring dismutation reaction of dye triplets with the ground state dye (the
Scheme I

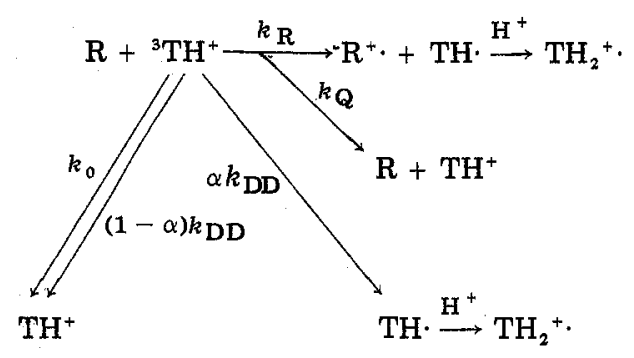

so-called DD process ${ }^{21}$ ). At constant concentration of the ground state this process may be considered to be quasi-monomolecular.

To calculate the radical yield for the basic triplet we made use of kinetic Scheme I. For the reactions of the acid triplet, according to eq 4, the protonation of the basic triplet is the first step. The acid triplet ${ }^{3} \mathrm{TH}_{2}{ }^{2+}$, thus produced, is then subject to the same reactions as the basic triplet in Scheme I. The schemes differ only in that the protonation occurs at the semithionine stage in the first case and at the triplet stage in the second case.

The rate constants in Scheme I refer to the following processes: $k_{0}$, monomolecular triplet decay to the ground state; $k_{\mathrm{DD}}$, quasi-monomolecular quenching by reaction of the triplet with ground state molecules (DD process); $\alpha$, fraction of radical formation in the DD process; $k_{\mathrm{R}}$, radical formation by the reaction with the donor; $k_{0}$, physical quenching by the donor. (It should be noted that it is kinetically equivalent whether the last two processes are treated as independent or as proceeding via a common intermediate.)

The hypothetical triplet concentration present after the flash if the triplet had infinite lifetime will be denoted $\left[{ }^{3} \mathrm{TH}^{+}\right]_{0}$. One obtains for $\left[\mathrm{TH}_{2}{ }^{+} \cdot\right]$, the acid semithionine concentration after the completed triplet reaction:

$\left[\mathrm{TH}_{2}^{+} \cdot\right]=\frac{\alpha k_{\mathrm{DD}}+k_{\mathrm{R}}[\mathrm{R}]}{k_{0}+k_{\mathrm{DD}}+\left(k_{\mathrm{R}}+k_{\mathrm{Q}}\right)[\mathrm{R}]}\left[{ }^{3} \mathrm{TH}^{+}\right]_{0}$

Writing

$x=\left[\mathrm{TH}_{2}^{+} \cdot\right] /\left[{ }^{3} \mathrm{TH}^{+}\right]_{0}$

$x_{0}=\alpha k_{\mathrm{DD}} /\left(k_{0}+k_{\mathrm{DD}}\right)$

$a=k_{\mathrm{R}} /\left(k_{0}+k_{\mathrm{DD}}\right)$

$b=\left(k_{\mathrm{R}}+k_{\mathrm{Q}}\right) /\left(k_{0}+k_{\mathrm{DD}}\right)$

one obtains

$x=\left(x_{0}+a[\mathrm{R}]\right) /(1+b[\mathrm{R}])$

or

$1 /\left(x-x_{0}\right)=b /\left(a-b x_{0}\right)+1 /\left(\left(a-b x_{0}\right)[R]\right)$

$1 /\left(x-x_{0}\right)$ plotted against $1 /[\mathrm{R}]$ must give a straight line. Denoting the semithionine absorption produced with a donor by $E$, and the corresponding quantity for donor free solutions by $E_{0}$, this linear relationship must hold also for $1 /\left(E-E_{0}\right)$ vs. $1 /[\mathrm{R}]$, because $E$ and $E_{0}$ are proportional to $x$ and $x_{0}$, respectively. Thus it is possible to extrapolate to $E_{\infty}$, i.e., $E$ for $[\mathrm{R}] \rightarrow \infty$. The half-quenching concentration of the donor $[R]_{1 / 2}$ can be obtained according to Figure 2. It is related to the triplet lifetime $\tau_{0}$ in quencher free solution by the equation:

$[\mathrm{R}]_{1 / 2}\left(k_{\mathrm{R}}+k_{\mathrm{Q}}\right)=k_{0}+k_{\mathrm{DD}}=1 / \tau_{0}$

From the measured value of the lifetime $\tau_{0}$ and the half-quenching concentration the sum of the quenching rate constants $\left(k_{\mathrm{R}}+k_{\mathrm{Q}}\right)$ can be calculated. This extrapolation procedure was applied to most of the sub- 


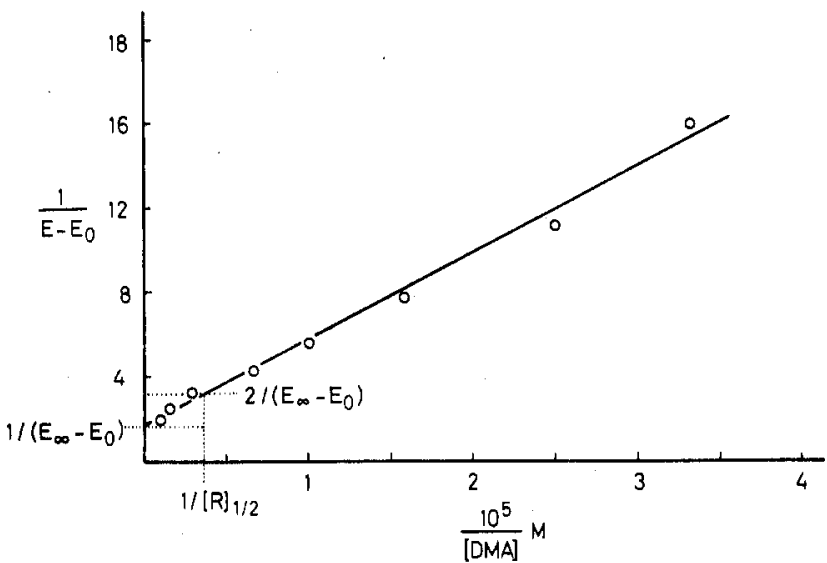

Flgure 2. Illustration of the extrapolation procedure for obtaining the semithionine absorption produced in the limiting case of infinite donor concentration. The determination of the half-quenching concentration from this plot is also shown. (Quencher DMA, $\left[\mathrm{TH}^{+}\right]=2 \times 10^{-5} \mathrm{M}$.)

stances. In the case of donors with radical yields of less than $30 \%$ this procedure could no longer be employed, since the measured changes of the quantity of semithionine produced varied too little. The sum of the quenching rate constants was then obtained directly from measurements of the triplet lifetime dependence on the donor concentration in nonbuffered solutions. With the known value of the quenching rate constant, determined by the latter method, donor concentrations could be chosen at which the limiting case of a quantitative reaction between triplet and donor was almost realized and the singlet reaction was still negligible. The acid semithionine absorption $E$ obtained in buffered solutions is related to $E_{\infty}$, the corresponding absorption for $[R] \rightarrow \infty$, by

$E_{\infty}=E+\left(E-E_{0}\right) \frac{1 / \tau_{0}}{\left(k_{\mathrm{R}}+k_{\mathrm{Q}}\right)[\mathrm{R}]}$

Absolute Values of the Quantum Yields. The absolute quantum yield of radical formation from the triplet state when the donor concentration approaches infinity is given by

$\eta_{\mathrm{R}}=\frac{k_{\mathrm{R}}}{k_{\mathrm{R}}+k_{\mathrm{Q}}}=\frac{\left[\mathrm{TH}_{2}^{+}\right]_{\infty}}{\left[{ }^{3} \mathrm{TH}^{+}\right]_{0}}=\frac{\epsilon^{\prime}}{\epsilon} \frac{E_{\infty}}{E_{0}{ }^{\prime}}$

$\left[\mathrm{TH}_{2}{ }^{+} \cdot\right]_{\infty}$ is the concentration of acid semithionine produced with donor concentrations extrapolated to infinity. $E_{0}{ }^{\prime}$ is the hypothetically obtainable triplet absorbance in the absence of any quenching process, and $\epsilon$ and $\epsilon^{\prime}$ are the molar extinction coefficients of semithionine and triplet, respectively. It was found convenient to carry out the determination of the absolute value of the limiting quantum yield $k_{\mathrm{R}} /\left(k_{\mathrm{Q}}+k_{\mathrm{R}}\right)$ for one standard substance with the basic triplet form and to compare the extrapolated values of the other donors with this standard. The standard substance chosen was DMA, because of its high radical yield and the stability of its solutions. $E_{0}^{\prime}$ was determined using solutions without quencher. The procedure is illustrated in Figure 3. The change of the optical density $E^{\prime}$ at $780 \mathrm{~nm}$ in quencher free solutions and with low flash intensity (negligible depletion of the ground state) is described by the differential equations:

$\mathrm{d} E_{\mathrm{T}} / \mathrm{d} t=c_{1} I(t)-c_{2} E_{\mathrm{T}}$

$\mathrm{d} E_{\mathrm{S}} / \mathrm{d} t=c_{3} E_{\mathrm{T}}$

and

$E^{\prime}=E_{\mathrm{T}}+E_{\mathrm{S}}$

where $I(t)$ is the flash intensity, given in arbitrary units
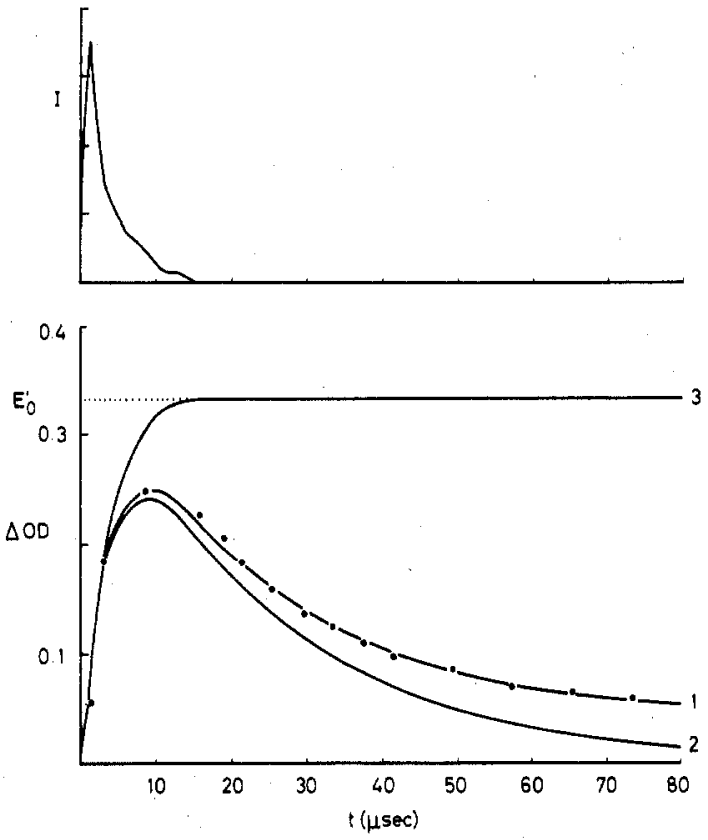

Figure 3. Determination of total concentration of triplets produced by a flash. $\triangle O D$ at $780 \mathrm{~nm}$ is plotted vs. time for a solution without quencher. (O) Experimental, $\left[\mathrm{TH}^{+}\right]=4 \times 10^{-5} \mathrm{M}$; computer simulation: curve $1, \triangle \mathrm{OD}$ due to triplet ${ }^{3} \mathrm{TH}^{+}$plus semithionine $\mathrm{TH}_{2}^{+} ;$; curve $2, \triangle \mathrm{OD}$ due to the triplet alone; curve $3, \triangle O D$ due to the triplet alone, if infinite lifetime is assumed. The upper part of the figure shows the time dependence of the flash intensity.

and obtained by recording the flash stray light. $E_{\mathrm{T}}$ and $E_{\mathrm{S}}$ are the optical densities due to the triplet or the semithionine, respectively. $c_{1}$ is a constant comprising the calibration factor of the arbitrary flash intensity as well as the extinction of the ground state and the geometry of the cuvette. $c_{2}$ and $c_{3}$ are constants which are related to the rate constants of Scheme I $\left(k_{0}+k_{\mathrm{DD}}\right), \alpha k_{\mathrm{DD}}$, and to the extinction coefficients of the species involved. By a proper choice of these constants the numerically calculated curve $E^{\prime}(t)$ can be fitted to the experimental one. $E_{0}^{\prime}$ is then obtain as the result of integrating eq 12 and setting $c_{2}=0$.

Second Method of Determination of Absolute Quantum Yields. The use of the extinction coefficients which are uncertain by an experimental error of $\pm 10 \%$ can be avoided if the disappearance of thionine from its ground state is observed directly and solutions with and without donor are compared. Since for such measurements the ground state has to be depopulated to a considerable extent, the condition of constant optical density for the absorption of the exciting flash light is no longer valid and hence the change in optical density no longer represents a linear measure of the quantum yield.

The best conditions for evaluating the quantum yield are given now in the limiting case of a small initial dye concentration, where there are only small optical densities for the absorption of the flash light. The pumping rate is then given by

$-\mathrm{d}\left[\mathrm{TH}^{+}\right] / \mathrm{d} t=j I(t)\left[\mathrm{TH}^{+}\right]$

incorporating a proportionality constant $j$ to be determined. The following two coupled differential equations result from Scheme I:

$$
\begin{aligned}
& \mathrm{d}\left[{ }^{3} \mathrm{TH}^{+}\right] / \mathrm{d} t=j I(t)\left[\mathrm{TH}^{+}\right] \\
& \quad-\left(k_{0}+\left(k_{\mathrm{Q}}+k_{\mathrm{R}}\right)[\mathrm{R}]\right)\left[{ }^{3} \mathrm{TH}^{+}\right] \\
& \mathrm{d}\left[\mathrm{TH}^{+}\right] / \mathrm{d} t=-j I(t)\left[\mathrm{TH}^{+}\right] \\
& \quad+\left(k_{0}+k_{\mathrm{Q}}[\mathrm{R}]\right)\left[{ }^{3} \mathrm{TH}^{+}\right]
\end{aligned}
$$


TABLE II: Total Quenching Constants $\left(k_{Q}+k_{R}\right)$ and Radical Yields $\left(\Phi_{R}=k_{R} /\left(k_{Q}+k_{R}\right)\right)$ of Different Electron Donors in Their Reactions with Basic and Acid Thionine Triplet ${ }^{3} \mathrm{TH}^{+}$and ${ }^{3} \mathrm{TH}_{2}{ }^{2+}$

\begin{tabular}{|c|c|c|c|c|}
\hline \multirow[b]{2}{*}{ Donors } & \multicolumn{2}{|c|}{ Reaction with ${ }^{3} \mathrm{TH}^{+}$} & \multicolumn{2}{|c|}{ Reaction with ${ }^{3} \mathrm{TH}_{2}{ }^{2+}$} \\
\hline & $\left(k_{\mathrm{Q}}+k_{\mathrm{R}}\right)^{a}$ & $\Phi_{\mathrm{R}}$ & $\left(k_{\mathbf{Q}}+k_{\mathbf{R}}\right)^{a}$ & $\Phi_{R}$ \\
\hline 9,10-Dimethylanthracene & $3.5 \times 10^{9}$ & $0.80 \pm 0.08$ & $4.0 \times 10^{9}$ & $0.80 \pm 0.08$ \\
\hline Azulene & $2.0 \times 10^{9}$ & $0.75 \pm 0.08$ & $4.0 \times 10^{9}$ & $0.85 \pm 0.09$ \\
\hline 1,3-Diphenylisobenzofuran & $4.5 \times 10^{9}$ & $0.80 \pm 0.08$ & $4.0 \times 10^{9}$ & $0.85 \pm 0.09$ \\
\hline 4-Methoxydimethylaniline & $5.0 \times 10^{9}$ & $0.80 \pm 0.1$ & & \\
\hline Hydroquinone & $2.5 \times 10^{9} \bar{b}$ & $0.85 \pm 0.09^{b}$ & $2.5 \times 10^{9}$ & $0.80 \pm 0.08$ \\
\hline Diazabicy clo[2.2.2] octane & $\left|8.0 \times 10^{8}\right|$ & $0.85 \pm 0.09$ & & \\
\hline 4-Bromoaniline & $3.0 \times 10^{9}$ & $0.40 \pm 0.05$ & $4.0 \times 10^{9}$ & $0.45 \pm 0.05$ \\
\hline Bromobenzene & $<4 \times 10^{4}$ & & $<4 \times 10^{4}$ & \\
\hline Thiourea & $7.5 \times 10^{6}$ & $0.20 \pm 0.03$ & $8.0 \times 10^{8}$ & $0.16 \pm 0.02$ \\
\hline $\mathrm{N}$-Allylthiourea & $8.0 \times 10^{6}$ & $0.31 \pm 0.03$ & $7.0 \times 10^{8}$ & $0.30 \pm 0.03$ \\
\hline Selenourea & $4.5 \times 10^{9}$ & $0.04 \pm 0.01$ & $3.5 \times 10^{9}$ & $0.10 \pm 0.02$ \\
\hline
\end{tabular}

${ }^{a}$ Units: $\mathrm{M}^{-1} \mathrm{~s}^{-1}$. ${ }^{b}$ Presumably hydrogen transfer.

$\left(k_{\mathrm{DD}}\right.$ is negligible at thionine concentrations as low as $10^{-6}$ $M)$ the numerical solutions of which can be fitted, by a suitable choice of the rate constant $k_{\mathrm{R}}$, to the experimentally obtained curve. (The rate constants $k_{0}$ and $\left(k_{\mathrm{R}}\right.$ $+k_{Q}$ ) can be obtained independently, from lifetime measurements and the half-quenching concentrations of the donors.) The constant $j$ is determined by a corresponding experiment without donor addition. The limiting yield of radical formation with infinite donor concentration is then given by $k_{\mathrm{R}} /\left(k_{\mathrm{R}}+k_{\mathrm{Q}}\right)$.

For values of $1 /\left(\left(k_{\mathrm{Q}}+k_{\mathrm{R}}\right)[\mathrm{R}]\right)$, short compared to the half-width of the flash, the evaluation may be simplified further. In this case the concentration of the triplet is well approximated by the quasi-stationary value

$\left[{ }^{3} \mathrm{TH}^{+}\right]=\frac{j I(t)\left[\mathrm{TH}^{+}\right]}{\left(k_{\mathrm{Q}}+k_{\mathrm{R}}\right)[\mathrm{R}]}$

This leaves one differential equation

$-\mathrm{d}\left[\mathrm{TH}^{+}\right] / \mathrm{d} t=\eta_{\mathrm{R}} j I(t)\left[\mathrm{TH}^{+}\right]$

yielding

$$
\begin{aligned}
\eta_{\mathbf{R}} & =\frac{\ln \left(\left[\mathrm{TH}^{+}\right]_{t=0} /\left[\mathrm{TH}^{+}\right]_{t=\infty}\right)}{j \int_{0}^{\infty} I(t) \mathrm{d} t}=\mathrm{constan} t \\
& \times \ln \frac{E(600)_{t=0}}{E(600)_{t=\infty}}
\end{aligned}
$$

Under these special conditions the quantity $\ln (E$ $\left.(600)_{t=0} / E(600)_{t=\infty}\right)$ represents a linear measure of the quantum yield required. ( $t=\infty$ means a long time as compared to the half duration of the flash, but a short time on the time scale of the semithionine decay. So in our case we had $t \approx 25 \mu \mathrm{s}$.) The advantage of this second method is that it works independently of the $\mathrm{pH}$ of the solution, as long as the ground state is not protonated or deprotonated. By means of this second method the same absolute value of radical formation from the triplet state was obtained as using the first method, DMA being the donor in both cases. In the case of donors giving low semithionine yields, this method clearly demonstrates, that the reduced yield of radicals is due to the bimolecular repopulation of the thionine ground state (Figure 4).

Special Properties of Some of the Donors. There were some problems due to the basic strength of some of the donors. $p$-Bromoaniline is a rather weak base which is not protonated by the methanolic buffer of $\mathrm{pH}^{*}$ 8.6. In the presence of $0.1 \mathrm{M}$ methanolic phosphoric acid the extent of protonation is near $50 \%$ at a $p$-bromoaniline concentration of $10^{-2} \mathrm{M}$ so that measurements of the reactivity and the quantum yield with the acid triplet were still possible. The effective concentration of unprotonated $p$-bromoaniline in each experiment was determined

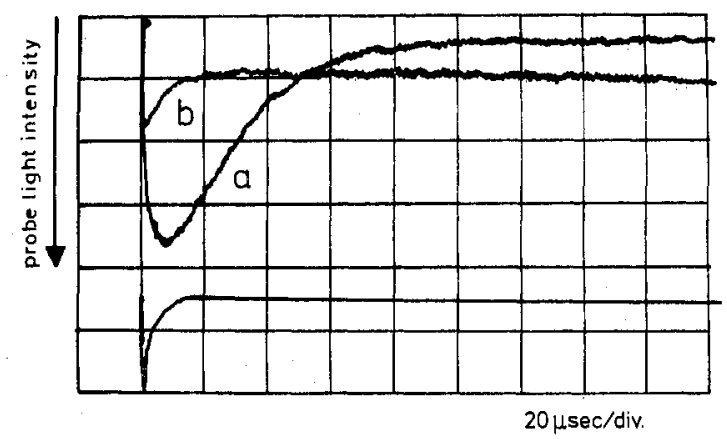

Figure 4. Demonstration of physical quenching of the thionine triplet ${ }^{3} \mathrm{TH}^{+}$by thiourea: (a) change in transparency at $600 \mathrm{~nm}$ (maximum of ground state absorption) due to repopulation of the ground state from the triplet state without thiourea; (b) the same measurement in the presence of $6.5 \times 10^{-2} \mathrm{M}$ thiourea. Flash intensity and thionine concentration $\left(10^{-6} \mathrm{M}\right)$ were the same for $a$ and $b$. The solvent was oxygen free methanol.

spectroscopically. The protonated form of $p$-bromoaniline shows no quenching of the thionine triplet ${ }^{3} \mathrm{TH}_{2}{ }^{2+}$, as could be shown by experiments with the completely protonated p-bromoaniline in the presence of $\mathrm{HCl}$.

The $\mathrm{p} K_{\mathrm{a}}{ }^{*}$ value of the singly protonated form of DABCO in methanol was determined by potentiometric titration with methanolic $\mathrm{HCl}$ to be 8.4. The buffer of $\mathrm{pH}^{*} 8.6$ may be used in this case, too, if the degree of protonation is taken into account. The reaction with the acid triplet, however, can no longer be measured. Also for $p$-methoxydimethylaniline only the reaction with the basic triplet could be studied, but the buffer of $\mathrm{pH}^{*} 8.6$ could not be used with this substance. The radical yield was determined by the second method. (The molar extinction coefficient of the donor radical amounts to less than 2000 at 600 $\mathrm{nm}^{22}$.)

\section{Results and Discussion}

The results for the triplet quenching constants (physical quenching plus radical formation) as well as the radical yields under conditions of complete triplet quenching are given in Table II. In general the quenching constants are of an order of magnitude expected for electron transfer reactions according to the results of Vogelmann et al. ${ }^{11}$ for similar systems. The rate constant of hydroquinone with the basic triplet is high, however, as compared to the methylated compound $p$-dimethoxybenzene $\left(10^{7} \mathrm{M}^{-1} \mathrm{~s}^{-1}\right){ }^{11}$ though the one-electron oxidation potential of hydroquinone certainly is not lower than that of $p$-dimethoxybenzene. A possible explanation might be that in the reaction of the basic triplet with hydroquinone a hydrogen transfer takes place, the free enthalpy of this reaction being more favorable than that for electron transfer. ${ }^{23}$ In fact 
Scheme II

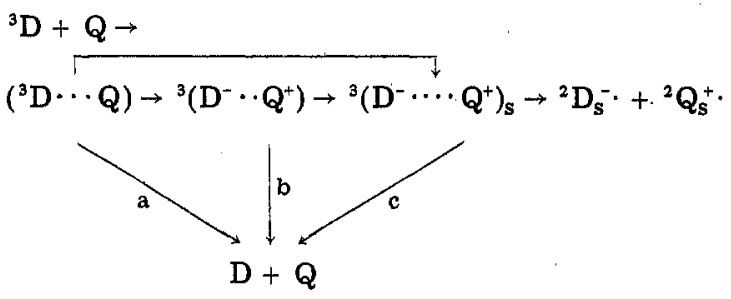

in nonbuffered methanol direct formation of acid semithionine $\mathrm{TH}_{2}^{+}$. can be observed.

The results demonstrate that for many of the electron donors, whose reactions with the thionine triplets have been studied, the radical yields are quite high. There are, however, compounds showing considerable or even dominant physical quenching. As the measured lifetime of the semireduced thionine is much longer than the flash duration, a recombination of free radicals during the first $20 \mu$ s after flash triggering can be ruled out as a possible cause of the decrease of radical yields. On the other hand, the experiments, excluding complex formation of the ground state molecules and quenching of the singlet excited thionine, confirm that electron transfer and physical quenching are dynamical processes of the thionine triplets. The question arises, whether both processes are independent or whether they are coupled in that they pass through a common intermediate.

The various possibilities are illustrated in Scheme II, analogous to the one used by Mataga. ${ }^{3 b}$ Here ${ }^{3} \mathrm{D}$ is the triplet excited dye molecule, $Q$ the quencher, $\left({ }^{3} \mathrm{D} \ldots \mathrm{Q}\right)$ is an encounter complex, ${ }^{3}\left(\mathrm{D}^{-} . . \mathrm{Q}^{+}\right)$is a triplet exciplex, ${ }^{3}\left(\mathrm{D}^{-} \ldots \mathrm{Q}^{+}\right)_{\mathrm{s}}$ the solvated radical pair, still in an overall triplet state, and ${ }^{2} \mathrm{D}^{-}$. and ${ }^{2} \mathrm{Q}^{+}$. are free radicals. The channel, denoted "reaction" in Mataga's scheme, is not important in our case, at least for the dye component, there is no fast chemical reaction other than radical formation.

Assuming first that the physical quenching is independent of electron transfer (reaction a), we have to consider triplet-triplet energy transfer and the external heavy atom effect as possible mechanisms.

Triplet-triplet energy transfer can be excluded as a substantial cause of triplet deactivation; when using azulene (triplet energy $38.6 \mathrm{kcal} / \mathrm{mol}^{24}$ ), the donor which probably has the lowest triplet energy and a short triplet lifetime, the radical yield is high. There may, however, be a small influence of triplet-triplet energy transfer: The conditions are more favorable in the case of the basic triplet form (triplet energy $39 \pm 1.5 \mathrm{kcal} / \mathrm{mol}^{14}$ ) than for the acid triplet form (triplet energy $30 \pm 2.5 \mathrm{kcal} / \mathrm{mol}^{14}$ ). In fact a slightly higher radical yield is found with the latter.

From Table II it can be seen that all donor compounds, exhibiting high physical quenching efficiency, contain heavy atoms or, in the case of the thioureas, at least atoms with moderate spin-orbit coupling constants. As is well known, intersystem crossing processes may be enhanced by external heavy atom perturbation. ${ }^{25}$ As was shown in fluorescence quenching experiments, the quenching efficiency of heavy atom quenchers decreases with increasing energy gap between the two coupling levels of different multiplicity in the fluorescing molecule. ${ }^{26}$ If this gap is larger than $30 \mathrm{kcal}$ much higher concentrations of the quencher are necessary than those involved in our experiments.

As may be learned from the comparison of $p$-bromoaniline with bromobenzene, the quenching efficiency of the former cannot be explained merely by the presence of the bromine atom. (There is no quenching of the thionine triplets with bromobenzene up to concentrations of $1 \mathrm{M}$.) Stronger evidence for an interrelation between electron transfer and the physical quenching reaction is given by the reactivity of thiourea and allylthiourea toward the different triplet forms of thionine. For these donors the radical yields with both triplet forms are the same, though the reaction with the acid triplet form is about 100 times faster than with the basic form. This means that the rate constants of both radical formation and physical quenching are increased by a factor of 100 , which strongly suggests that the physical quenching follows the electron transfer step, the latter being rate determining. In order to explain the physical quenching one should therefore discuss an intersystem crossing process in the primarily formed electron transfer product, which competes with the formation of free radicals (b or c, Scheme II). ${ }^{27}$

Triplet exciplexes, if formed in electron transfer reactions, are not expected to be detectable by their phosphorescence in fluid solutions. Spectroscopic evidence has been given for triplet exciplexes in nonpolar solvents, ${ }^{28}$ in polar solvents, however, their absorption spectra are not likely to be very different from the radical ion spectra. ${ }^{29}$ From this point of view it would be of value to obtain some other means of probing triplet exciplexes in electron transfer reactions in polar solvents.

Intersystem crossing processes in the exciplex are often faster than in the excited single component. ${ }^{30}$ This may in part be due to the fact that symmetry conditions are less restrictive for the intermolecular spin-orbit coupling matrix elements. Enhanced spin-orbit coupling should be expected when heavy atoms are introduced. This was verified by Gronkiewicz et $\mathrm{al}^{30}$ in the case of phosphorescing CT complexes. An exciplex model for the electron transfer reaction in our systems could explain the smaller radical yield with $p$-bromoaniline as compared to the other aromatics and of selenourea as compared to the thioureas.

Exciplexes can dissociate to form pairs of individually solvated radical ions. The electronic interaction of the geminate radicals decreases exponentially with their distance. ${ }^{31}$ This may be seen, e.g., by the singlet-triplet splitting of the radical pair. ${ }^{32}$ However, though the lowest electronic singlet and triplet state of the radical pair may become completely degenerate, the multiplicity of the reaction complex is retained in the resulting radical pair for a period of time which may be determined by various mechanisms. Spin-lattice relaxation is usually too slow to become important during the geminate phase of the radical pair in solutions of normal viscosity. ${ }^{33}$ As was shown by Kaptein ${ }^{34}$ for radical pairs with orbitally nondegenerate ground states the influence of spin-orbit coupling can also be neglected. Thus the heavy atom effect we observed favors the exciplex as the electron transfer intermediate, where the intersystem crossing takes place, rather than the radical pair.

Very recently it has been demonstrated by the magnetic field dependence of fast triplet formation from geminate radical pairs, produced by electron transfer from aromatic amines to singlet excited pyrene, that intersystem crossing in radical pairs can be due to the hyperfine coupling between electronic and nuclear spins. ${ }^{35,36}$ The same mechanism might be operative in the intersystem crossing of radical pairs, originally formed in the electronic triplet state, making a recombination to singlet ground state products possible.

High rate constants would be expected for the recombination of the semithionine with the oxidized donor radicals (yielding the ground state molecules) due to the negative free enthalpy of these reactions. The long lifetime 
observed for the semithionine radicals is not consistent with such a high recombination rate constant of counterradicals, however, unless it is assumed that the concentration of the oxidized counterradicals is diminished by some other process (e.g., involving the solvent). Such a process might be fast enough to prevent the recombination of freely diffusing radicals, but slow enough not to interfere with the geminate recombination phase of the radicals.

The reported yields of geminate recombination products due to hyperfine induced intersystem crossing are about $10 \%{ }^{35}$ They are comparable to the small physical quenching yields of the donors in the upper half of Table I. It is doubtful, however, whether the hyperfine mechanism can account for the large intersystem crossing yields, which we have observed with some donors. The radicals of the thioureas and selenourea are expected to contribute less to the hyperfine coupling than the aromatic donor cations, as the radical electron is localized at atoms ( $\mathbf{S}$ or Se) having nuclear spin zero for the major part of their natural isotopes. Furthermore in the radical pairs of our systems the diffusive separation of the radicals is not hindered by Coulomb attraction.

In order to obtain more conclusive information, experiments are in progress to test the magnetic field dependence of the radical yield in electron transfer reactions of triplet states.

Acknowledgment. We are indebted to Professor A. Weller and Dr. M. E. Michel-Beyerle for making preprints of their papers available to us. The help of D. Miller in translating the manuscript is gratefully acknowledged. We thank the Fonds der Chemischen Industrie and the Deutsche Forschungsgemeinschaft for their financial assistance.

\section{References and Notes}

(1) H. Leonhardt and A. Weller, Z. Phys. Chem. (Frankfurt am Main) 29, 277 (1961); "Luminescence of Organic and Inorganic Materials", H. P. Kallmann and G. M. Spruch, Ed., Wiley, New York, N.Y., 1962 p 74.

(2) For references see ref $3 a$.

(3) (a) H. Masuhara, T. Hino, and N. Mataga, J. Phys. Chem., 79, 994 (1975); (b) T. Hino, H. Akazawa, H. Masuhara, and N. Mataga, ibid., $80,33(1976)$.

(4) N. Orbach and M. Ottolenghi in "The Exciplex", M. Gordon and W. R. Ware, Ed., Academlc Press, New York, N.Y, 1975, p 75

(5) K. H. Grellmann, A. R. Watkins, and A. Weller, J. Lumin., 1,2, 678 $(1970)$

(6) H. Fischer, Z. Phys. Chem. (Frankfurt am Main), 43, 177 (1964).
(7) H. Fischer, H.'E. A. Kramer, and A. Maute, Z. Phys. Chem. (Frankfurt am Main), 69," 113 (1970).

(8) J. Faure, R. Bonneau, and J. Joussot-Dublen, Photochem. Photoblol., 6, 331 (1967); R. Bonneau and J. Joussot-Dublen, lbid., 17, 313 (1973).

(9) H. E. A. Kramer and A. Maute, Photochem. Photobiol., 15, 7 (1972).

(10) E. Vogeimann and H. E. A. Kramer, Photochem. Photobiol., 23, 383 (1976).

(11) E. Vogelmann, S. Schreiner, W. Rauscher, and H. E. A. Kramer, Z. Phys. Chem. (Frankfurt am Main), 101, 321 (1976).

(12) D. Rehm and A. Weller, Ber. Bunsenges. Phys, Chem., 73, 834 (1969).

(13) E. Vogelmann, H. Schmidt, U. Steiner, and H. E. A. Kramer, Z. Phys. Chem. (Frankfurt am Main), 94, 101 (1975).

(14) H. E. A. Krather, Z. Phys. Chem. (Frankfurt am Main), 66, 73 (1969).

(15) W. Endriss; Thesis, University of Stuttgart, 1961.

(16) D. G. Thómas, J. H. Billman, and C. E. Davis, J. Am. Chem. Soc., $68,895(1946)$

(17) C. L. De Ligny, P. F. M. Luykx, M. Rehbach, and A. A: Wieneke, Recl. Trav. Chim. Pays-Bas, 79, 699 (1960)

(18) W. Broser and H. Fleischhauer, Z. Naturforsch. B, 25, 1389 (1970).

(19) U. Steiner, M. Hafner, S. Schreiner, and H. E. A. Kramer, Photochem. Photobiol., 19, 119'(1974).

(20) A. Kira, S. Arai, and M. Imamura, J. Phys. Chem., 76, 1119 (1972).

(21) (a) L. Lindqvist, Ark. 'Kem., 16, 79 (1960); (b) N.'Kosul, K. Uchida, and M. Kolzumi, Bull. Chem. Soc. Jpn, 38, 1958 (1965)

(22) W. Atchele, Diploniarbelt 1975, University of Stuttgart.

(23) D. Rehm and A. Weller, Isr. J. Chem., 8, 259 (1970)

(24) (a) P. Kröning, Z. Phys. Chem. (Frankfurt am Main), 86, 225 (1973); (b) W: G. Herksthoeter, J. Am. Chem. Soc., 97, 4161 (1975).

(25) S. P. McGlynn, T. Azumi, and M. Kinoshita, "Molecular Spectroscopy of the Triplet State", Prentice-Hall, Englewood Cllffs, N.J., 1969.

(26) H. Dreeskamp and M: Zander, Z. Naturforsch. A, 28, 1743 (1973); H. Dreeskamp, E. Koch, and M. Zander, Chem. Phys. Lett., 31, 251 (1975).

(27) After our manuscript had been written, we saw the recent paper of A. Treinin and E. Hayon, J. Am. Chem. Soc., 98, 3884 (1976), on the quenching of triplet states by inorganic ions. Their results, too, Indicate that chaige transier has to occur to make the intersystem crossing process fossible. In contrast to our results, however, with inorganic tons as quenchers no formation of radicals can be observed (except in one case), which these authors explain by the higher effectivity of the intersystem crossing process as compared to the dissociation of the CT complex

(28) D. G. Whitten, J. K. Roy, and F. A. Carroll, in ref 4, p 247.

(29) N. Orbach and M. Ottolenghi, Chem. Phys. Lett., 35, 175 (1975).

(30) M. Gronkiewicz; B. Kozankiewicz, and J. Prochorow, Chem. Phys. Lett., 38, 325 (1976)

(31) F. Adrian, J..Chem. Phys., 57, 5107 (1972).

(32) K. Itoh, H. Hayashi, and S. Nagakura, Mol. Phys., 17, 561 (1969).

(33) B. Brocklehurst, Nature (London), 221, 921 (1969).

(34) R. Kaptein, J. Am. Chem. Soc., 94, 6251 (1972).

(35) K. Schulten, H. Staerk, A. Weller, H.-J. Werner, and B. Nickel, Z. Phys. Chem. (Frankfurt am Maln), 101, 371 (1976).

(36) R. Haberkorn and M. E. Michel-Beyerle, Z. Naturforsch. A, 31, 499 (1976); M. E. Michel-Beyerle, R. Haberkorn, W. Bube, E. Steffens, H. Schröder, H. J. Neusser, E. W. Schlag, and H. Seidlitz, Chem. Phys., 17, 139 (1976).

(37) Note Added in Proof: Meanwhile the lifetime of the first excited singlet state of thionine has been determined experimentally to be $3.6 \times$ $10^{-10} \mathrm{~s}$ at a concentration of $2.5 \times 10^{-4} \mathrm{M}$ in methanol. (E. Vogelmann, M. Hauser, and G. Heidt, private communication.) 